\title{
ANÁLISE DA ROTULAGEM DE LEITE EM PÓ INTEGRAL EM RELAÇÃO AOS CRITÉRIOS EXIGIDOS PELAS LEGISLAÇÕES VIGENTES
}

\section{ANALYSIS OF WHOLE POWDER MILK LABELING IN RELATION TO THE CRITERIA REQUIRED BY CURRENT LEGISLATION}

\author{
Caroline Aparecida Mazocco ${ }^{\mathbb{D}}$, Julie Facchini ${ }^{\mathbb{D}}$, Patricia Fassina* ${ }^{*}$ \\ Universidade do Vale do Taquari, Lajeado, Rio Grande do Sul, Brasil. \\ *patriciafassina@univates.br
}

\section{RESUMO}

O Brasil está entre os principais países produtores de leite do mundo. Com o passar dos anos, além da comercialização do leite fluído, a tecnologia conseguiu transformar o líquido em pó através de um processo de evaporação e dessecação que mantém o seu valor nutricional e impede o crescimento de microrganismos deteriorantes e patogênicos. O rótulo do leite em pó, assim como todos os alimentos embalados, deve conter informações obrigatórias que as legislações específicas exigem para manter a comunicação e a segurança alimentar e nutricional do consumidor. O presente estudo objetivou analisar a rotulagem de leite em pó integral produzidos no Vale do Taquari-RS e comercializados em supermercados de uma cidade da região a fim de comparar com os critérios exigidos pelas legislações em vigor. Para a escolha dos produtos foram definidos dois parâmetros, sendo eles embalagem de $400 \mathrm{~g}$ e leite em pó do tipo integral por ter maior disponibilidade nos mercados visitados. Foram analisados os rótulos tanto para os critérios de rotulagem geral quanto para aqueles de rotulagem nutricional, tendo como base as legislações vigentes que regulamentam a rotulagem de alimentos no Brasil, sendo elas a RDC n ${ }^{\circ}$ 259/2002, RDC no 359/2003 e RDC n 360/2003. Verificou-se que em todos os quesitos avaliados os rótulos estavam em conformidade com as legislações brasileiras vigentes. Ainda, foi possível demonstrar que as indústrias desta região estão seguindo corretamente as normas e especificações que a legislações brasileiras vigentes impõem quanto aos critérios de rotulagem de alimentos.

Palavras-chave: Embalagem de alimentos. Legislação sobre alimentos. Leite em pó. Rotulagem de alimentos.

\section{ABSTRACT}

Brazil is among the main milk producing countries in the world. Over the years, besides the commercialization of fluid milk, technology has managed to transform the liquid into powder through an evaporation and desiccation process, which maintains its nutritional value and prevents the growth of deteriorating and pathogenic microorganisms. The label for powder milk, as well as all packaged foods, must contain mandatory information required by specific legislation to maintain communication, nutritional and food security for the consumer. This study aimed to analyze the labeling of whole powdered milk produced in Vale do Taquari-RS and sold in supermarkets from a city in this region in order to compare it with the criteria required by the current legislation. In order to choose the products, we defined two parameters such as $400 \mathrm{~g}$ packaging and whole powder milk because it has greater availability in the visited markets. We analyzed labels for both general and nutritional labeling criteria based on the current laws that regulate food labeling in Brazil, which are RDC no. 259/2002; RDC no. 359/2003; and RDC no. 360/2003. We also found that the labels were in compliance with the current Brazilian legislation in all evaluated items. In addition, it was possible to demonstrate that industries in the mentioned region are correctly following the standards and specifications imposed by Brazilian current legislation regarding food labeling criteria.

Keywords: Food packaging. Food legislation. Powdered milk. Food labeling. 


\section{INTRODUÇÃO}

As indústrias brasileiras de alimentos e bebidas são essenciais e de grande comprometimento na sua produção e abastecimento, pois através delas os alimentos são disponibilizados com qualidade e segurança alimentar em todos os continentes do planeta. Os alimentos produzidos são comercializados em mais de 180 países, tornando o Brasil o segundo maior exportador de alimentos industrializados do mundo. Em 2019, o faturamento das indústrias representou 9,6\% do Produto Interno Bruto (PIB) no país (ABIA, 2019).

Em relação à produção e comercialização de leite, no mundo, a União Europeia, os Estados Unidos e o Brasil são os principais países produtores e, em 2017, a produção cresceu, aproximadamente, 6 bilhões de litros. Segundo o Instituto Brasileiro de Geografia e Estatística (IBGE), no Brasil, a produção de leite de vaca cresceu em 2019, chegando a 34,8 bilhões de litros, um aumento de 2,7\% em relação ao ano anterior. $\mathrm{O}$ valor de produção atingiu $\mathrm{R} \$ 43,1$ bilhões, sendo Minas Gerais o maior estado produtor, seguido por Paraná e Rio Grande do Sul. Em 2019, o valor de produção dos principais produtos pecuários cresceu 9,0\%, somando $\mathrm{R} \$ 59,3$ bilhões, sendo 72,8\% desse valor só de produção de leite (IBGE, 2021). Já em relação ao consumo per capita de leite, em 2018, o Brasil alcançou 166,4 litros/habitante (SIQUEIRA, 2019).

Com as tecnologias presentes nas indústrias, o leite fluido é facilmente transformado em pó cuja finalidade é de aumentar a sua vida útil de prateleira, prevenindo o desenvolvimento de microrganismos, devido à redução do teor de água, diminuir as alterações nutritivas e sensoriais, garantindo a conservação dos nutrientes e possibilitar o seu transporte mais econômico (OLIVEIRA; MARCHIORE, 2017). Para isso, o leite é pasteurizado a fim de eliminar a maioria de patógenos e deteriorantes, sendo posteriormente concentrado até atingir, aproximadamente, $50 \%$ dos sólidos totais. Após, segue para o processo de evaporação, onde o líquido e o vapor formados se desprendem, sendo a etapa de secagem a última que transforma o leite fluido em uma fina camada de pó. Assim, o pó é removido para um moinho de martelo que converte as partículas em tamanhos uniformes seguindo para a embalagem. Entretanto, o processo mais utilizado nas indústrias para secagem do leite é o processo por Spray-dryer, composto por secadores com jato ou bico ou por atomizadores rotatórios que permitem retirar o pó, resfriá-lo e embalá-lo (IWASE, 2017).

Assim, "entende-se por leite em pó o produto que se obtém por desidratação do leite de vaca, integral, desnatado ou parcialmente desnatado e apto para a alimentação humana, mediante processos tecnologicamente adequados" (BRASIL, 2018). Este produto tem lugar de destaque na alimentação da população brasileira e, por consequência, tem grande importância no cenário econômico do País. Fazendo uma análise estadual, Santa Catarina, São Paulo, Paraná, Rio Grande do Sul e Goiás representam os seis Estados de maior produção de leite em pó no Brasil, sendo responsáveis pelo aumento da produção nacional, respondendo a cerca de $84,02 \%$ da produção no País (CARVALHO; ROCHA; GOMES, 2018).

Nos tempos modernos e agitados em que vivemos, uma grande parte da população busca por alimentos práticos, de fácil preparo e nutritivos. Com o avanço das tecnologias é possível que o leite em pó seja produzido com as características idênticas ao leite em seu estado natural, mantendo o teor de carboidratos, proteínas, gorduras e minerais, como o sódio e o cálcio (GREGÓRIO et al., 2020). Os elementos que compõem um produto devem ser descritos nos rótulos das embalagens para auxiliar o consumidor no entendimento quanto aos ingredientes e componentes nutricionais que o integram (SANTOS et al., 2017). Atualmente, os rótulos e as embalagens vêm se destacando com cores e formas, atraindo os consumidores, porém estes estão mais atentos às informações que eles carregam das indústrias, constituindo um meio de comunicação entre ambos (ANVISA, 2020). Assim, o rótulo de um produto é o primeiro contato entre o consumidor e o fornecedor no instante da compra (SILVA et al., 2017).

Os rótulos de leite em pó devem conter informações obrigatórias por legislações específicas para que os alimentos sejam seguros aos consumidores, sendo que estas não podem estar incompletas, conter incorreções ou induzir o consumidor ao erro (ANVISA, 2020). Dentre as legislações 
específicas para a rotulagem de alimentos consta a RDC n ${ }^{\circ} 259$, de 20 de setembro de 2002, que regulamenta a rotulagem de alimentos comercializados, independente da sua origem, embalados na ausência do cliente e prontos para serem fornecidos aos consumidores, visando o aperfeiçoamento do controle sanitário na área de alimentos com o objetivo de proteger à saúde da população. Essa previsão legal obriga os rótulos a apresentarem o idioma oficial do país, a identificação da origem e do lote, o prazo de validade, o conteúdo líquido, a lista de ingredientes, a declaração de aditivos, as instruções sobre o preparo antes do consumo, quando necessário, entre outros (BRASIL, 2002).

Outra legislação vigente é a RDC n 360, de 23 de dezembro de 2003, que regulamenta sobre a rotulagem nutricional dos alimentos embalados, tornando-a obrigatória. Nela, deve constar a declaração nutricional obrigatória de valor energético, carboidratos, proteínas, gorduras totais, gorduras saturadas, gorduras trans, fibra alimentar e sódio, de forma a auxiliar o consumidor a conhecer e escolher os alimentos com propriedades nutricionais que lhe agregam e contribuem em benefício da sua saúde (BRASIL, 2003b). Para complementar esta legislação a RDC no 359, de 23 de dezembro de 2003 estabelece os tamanhos das porções dos alimentos embalados para fins de rotulagem nutricional. Em vista disso, o objetivo do presente estudo foi analisar a rotulagem de leites em pó integral produzidos no Vale do Taquari-RS e comercializados em supermercados de uma cidade da região a fim de comparar com os critérios exigidos pelas legislações em vigor (BRASIL, 2003a).

\section{MATERIAL E MÉTODOS}

Estudo de caráter quantitativo, descritivo, exploratório e observacional para o qual foram analisadas sete diferentes marcas de leite em pó produzidas no Vale do Taquari-RS, comercializadas em todo o Brasil, mas que, para este estudo, foram adquiridas em supermercados de uma cidade da região. A escolha das marcas foi feita aleatoriamente, sendo baseada na disponibilidade das mesmas nos estabelecimentos visitados entre os meses de junho e julho de 2020. Para a escolha dos produtos foram definidos dois parâmetros, sendo eles embalagem de $400 \mathrm{~g}$ e leite em pó do tipo integral, por terem maior disponibilidade nos locais avaliados.

Os rótulos das embalagens foram analisados tendo como base as legislações vigentes que regulamentam a rotulagem de alimentos no Brasil, sendo elas a RDC nº 259/2002 e RDC no 359/360. Assim, foram avaliados os critérios obrigatórios que devem constar nas rotulagens dos alimentos, segundo a RDC nº 259/2002 (Quadro 1).

Quadro 1 - Critérios obrigatórios que devem constar nas rotulagens dos alimentos, segundo a RDC $\mathrm{n}^{\circ} 259 / 2002$

\section{Critérios de avaliação da rotulagem do leite em pó}

1. Lista de ingredientes (precedida da expressão "ingredientes" ou “ingr.”).

2. Declaração de aditivo (nome completo ou número INS - Sistema Internacional de Numeração ou Codex Alimentarius FAO/OMS).

3. Identificação de origem (precedida da expressão "fabricado em", "produto" ou "indústria").

4. Identificação do lote (precedida da expressão "L").

5. Prazo de Validade (precedida da expressão "válido até", "validade", "val:", "vence", "vencimento", "vto", "venc:", "consumir antes de", "consumir preferencialmente antes de").

6. Preparo e Instruções de Uso (instruções sobre o modo apropriado de uso, incluído a reconstituição).

7. Conservação do Produto e Tamanho da letra (não podendo ser inferior a $1 \mathrm{~mm}$ ).

Fonte: adaptado da RDC n ${ }^{\circ}$ 259/2002 (BRASIL, 2002). 
Ainda, foi avaliada a rotulagem nutricional segundo os critérios obrigatórios da RDC n³60/2003 (Quadro 2).

Quadro 2 - Critérios obrigatórios que devem constar nas rotulagens dos alimentos, segundo a RDC $\mathrm{n}^{\circ} 360 / 2003$

\section{Critérios de avaliação da rotulagem do leite em pó}

1. Valor nutricional:

- Valor Energético (kcal), Carboidratos (g), Proteínas (g), Gorduras Totais (g), Gorduras Saturadas (g), Gorduras Trans (g), Fibra Alimentar (g) e Sódio (mg).

2. Porção $(\mathrm{g})^{*}$ do alimento para fins de rotulagem nutricional.

3. Expressão dos valores da rotulagem nutricional:

- Valor Energético e o percentual de Valor Diário (\% VD) devem ser declarados em números inteiros;

- Nutrientes que possuem valores maiores ou iguais a 100 devem ser declarados em números inteiros com três cifras;

- Valores menores que 100 e maiores ou iguais a 10 devem ser declarados em números inteiros com duas cifras;

- Valores menores que 10 e maiores ou iguais a 1 devem ser declarados com uma cifra decimal;

- Valores menores que 1, para vitaminas e minerais, devem ser declarados com duas cifras decimais e os demais nutrientes devem ser declarados com uma cifra decimal.

Fonte: Adaptado da RDC n 259/2002 (BRASIL, 2002). *A porção foi analisada conforme a RDC n 359/2003 (BRASIL, 2003a).

Além disso, pode-se declarar como "0" ou "zero" ou "não contém" para o valor energético e ou nutrientes quando o alimento contiver quantidades menores ou iguais as estabelecidas como "não significativas", ou seja, quando os valores forem menores ou iguais a 4 kcal para o valor energético, menor ou igual a $0,5 \mathrm{~g}$ para carboidratos, menor ou igual a $0,5 \mathrm{~g}$ para proteínas, menor ou igual a 0,5 g para gorduras totais, menor ou igual a 0,2 g para gorduras saturadas, menor ou igual a $0,2 \mathrm{~g}$ para gorduras trans, menor ou igual a $0,5 \mathrm{~g}$ para fibra alimentar e menor ou igual a $5 \mathrm{mg}$ para sódio.

Para o presente estudo, as rotulagens das embalagens analisadas foram identificadas com letras (A, B, C, D, E, F, G) para manter o sigilo das marcas. Os resultados foram apresentados em tabelas / quadros, sendo uma relacionada à caracterização da informação nutricional dos rótulos das amostras de leite em pó integral analisadas, em valores de média e desvio padrão, e duas comparativas frente às rotulagens das diferentes marcas do produto, sendo a primeira relacionada aos critérios obrigatórios, conforme a RDC n ${ }^{\circ}$ 259/2002, e a outra segundo a informação nutricional do alimento, conforme a RDC n $n^{\circ} 359 / 2003$ e RDC $n^{\circ} 360 / 2003$, nas quais os parâmetros foram avaliados em Conforme (C) e Não conforme (NC).

\section{RESULTADOS E DISCUSSÃO}

No Quadro 3 encontram-se as informações relacionadas aos critérios obrigatórios constantes nos rótulos das amostras de leite em pó integral analisadas, segundo exigência da RDC nº 259/2002. Observou-se que todas as amostras de leite em pó estavam em conformidade, atendendo aos requisitos exigidos pela legislação vigente.

A Tabela 1 apresenta a caracterização da informação nutricional contida nos rótulos das amostras de leite em pó integral analisadas segundo os critérios obrigatórios da RDC n 359/2003 e 
RDC $n^{\circ} 360 / 2003$, em média e desvio padrão, e as expressões dos valores da rotulagem nutricional, conforme a RDC n ${ }^{\circ}$ 360/2003. Tratando-se de Porção, Carboidratos, Gordura Trans e Fibra Alimentar verificou-se que os valores apresentados foram homogêneos, ou seja, este conjunto de dados apresentou-se de forma uniforme em todos os rótulos analisados, sendo a Porção de $26 \mathrm{~g}$, o teor de Carboidratos de $10 \mathrm{~g}$, não contendo Gordura Trans e Fibra Alimentar. Porém, os valores de Valor Energético, Proteínas, Gorduras Totais, Gorduras Saturadas e Sódio apresentaram pequenas

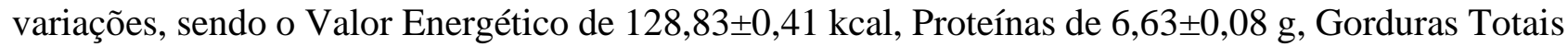

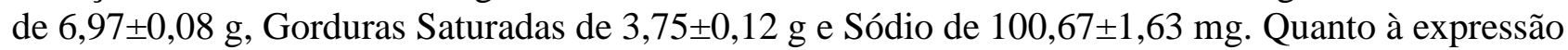
dos valores da rotulagem nutricional, todas as amostras de leite em pó integral avaliadas apresentaram conformidade com os critérios da legislação.

Quadro 3 - Conformidade das informações relacionadas aos critérios obrigatórios constantes nos rótulos das amostras de leite em pó integral analisadas, segundo a RDC no 259/2002

\begin{tabular}{|c|c|c|c|c|c|c|c|}
\hline \multirow{2}{*}{$\begin{array}{c}\text { Critérios obrigatórios da } \\
\text { rotulagem de alimentos }\end{array}$} & $\mathbf{A}$ & $\mathbf{B}$ & $\mathbf{C}$ & $\mathbf{D}$ & $\mathbf{E}$ & $\mathbf{F}$ & $\mathbf{G}$ \\
\hline Lista de Ingredientes & $\mathrm{C}$ & $\mathrm{C}$ & $\mathrm{C}$ & $\mathrm{C}$ & $\mathrm{C}$ & $\mathrm{C}$ & $\mathrm{C}$ \\
\hline Declaração de Aditivos (se houver) & $\mathrm{C}$ & $\mathrm{C}$ & $\mathrm{C}$ & $\mathrm{C}$ & $\mathrm{C}$ & $\mathrm{C}$ & $\mathrm{C}$ \\
\hline Identificação de Origem & $\mathrm{C}$ & $\mathrm{C}$ & $\mathrm{C}$ & $\mathrm{C}$ & $\mathrm{C}$ & $\mathrm{C}$ & $\mathrm{C}$ \\
\hline Identificação do Lote & $\mathrm{C}$ & $\mathrm{C}$ & $\mathrm{C}$ & $\mathrm{C}$ & $\mathrm{C}$ & $\mathrm{C}$ & $\mathrm{C}$ \\
\hline Prazo de Validade & $\mathrm{C}$ & $\mathrm{C}$ & $\mathrm{C}$ & $\mathrm{C}$ & $\mathrm{C}$ & $\mathrm{C}$ & $\mathrm{C}$ \\
\hline Preparo e Instruções de Uso & $\mathrm{C}$ & $\mathrm{C}$ & $\mathrm{C}$ & $\mathrm{C}$ & $\mathrm{C}$ & $\mathrm{C}$ & $\mathrm{C}$ \\
\hline Conservação do Produto & $\mathrm{C}$ & $\mathrm{C}$ & $\mathrm{C}$ & $\mathrm{C}$ & $\mathrm{C}$ & $\mathrm{C}$ & $\mathrm{C}$ \\
\hline Tamanho da Letra & $\mathrm{C}$ & $\mathrm{C}$ & $\mathrm{C}$ & $\mathrm{C}$ & $\mathrm{C}$ & $\mathrm{C}$ & $\mathrm{C}$ \\
\hline
\end{tabular}

Notas: $\mathrm{C}=$ Conforme.

Fonte: os autores.

Tabela 1 - Caracterização da informação nutricional dos rótulos das amostras de leite em pó integral analisadas segundo os critérios obrigatórios da RDC n ${ }^{\circ} 359 / 2003$ e RDC n ${ }^{\circ} 360 / 2003$, em valores de média e desvio padrão, e avaliação das expressões dos valores da rotulagem nutricional, conforme a $\mathrm{RDC} \mathrm{n}^{\circ} 360 / 2003$

\begin{tabular}{lcccccccc}
\hline \multirow{2}{*}{$\begin{array}{l}\text { Critérios obrigatórios da } \\
\text { informação nutricional }\end{array}$} & A & B & C & D & E & F & Média & DP \\
\cline { 2 - 7 } & 26 & 26 & 26 & 26 & 26 & 26 & 26,00 & 0,00 \\
Porção (g) & 129 & 129 & 129 & 128 & 129 & 129 & 128,83 & 0,41 \\
Valor Energético (kcal) & 10 & 10 & 10 & 10 & 10 & 10 & 10,00 & 0,00 \\
Carboidratos (g) & 6,6 & 6,6 & 6,6 & 6,8 & 6,6 & 6,6 & 6,63 & 0,08 \\
Proteínas (g) & 7,0 & 7,0 & 7,0 & 6,8 & 7,0 & 7,0 & 6,97 & 0,08 \\
Gorduras Totais (g) & 3,7 & 3,7 & 3,7 & 4,0 & 3,7 & 3,7 & 3,75 & 0,12 \\
Gorduras Saturadas (g) & 0 & 0 & 0 & 0 & 0 & 0 & 0,00 & 0,00 \\
Gorduras Trans (g) & 0 & 0 & 0 & 0 & 0 & 0 & 0,00 & 0,00 \\
Fibra Alimentar (g) & 100 & 100 & 100 & 104 & 100 & 100 & 100,67 & 1,63 \\
Sódio (mg) & & & & & &
\end{tabular}

Notas: $\mathrm{DP}=$ desvio padrão; $\mathrm{g}$ = grama; $\mathrm{mg}$ = miligrama; $\mathrm{kcal}=$ quilocaloria.

Fonte: os autores.

No Quadro 4 encontram-se as conformidades das informações nutricionais obrigatórias constantes nos rótulos das amostras de leite em pó integral analisadas conforme exigência da RDC 360/2003. Verificou-se que todas as amostras de leite em pó estavam em conformidade, atendendo aos requisitos exigidos pela legislação vigente.

Todas as seis amostras analisadas no presente estudo quanto aos critérios obrigatórios da rotulagem de alimentos, conforme a $\mathrm{RDC} \mathrm{n}^{\circ}$ 259/2002, apresentaram-se de acordo com a legislação. O mesmo resultado foi encontrado por Silva et al. (2017) ao analisarem oito rótulos de leite em pó 
integral de diferentes marcas comercializadas na cidade de Garanhuns-PE. Da mesma forma, Gregório et al. (2020), ao analisarem 5 diferentes marcas de leite em pó comercializados na cidade de Acari-RN também verificaram conformidade com os critérios obrigatórios exigidos pela legislação na rotulagem destes alimentos. Porém, diferente do atual estudo, Silva Filho, Araújo e Morais (2015), ao analisarem os rótulos de leite UHT comercializados na cidade de João Pessoa-PB, encontraram duas amostras sem lote, não estando de acordo com a legislação vigente.

Quadro 4 - Conformidade das informações nutricionais obrigatórias exigidas pela RDC nº 360/2003 em relação às amostras de leite em pó integral analisadas

\begin{tabular}{|l|c|c|c|c|c|c|}
\hline \multirow{2}{*}{$\begin{array}{l}\text { Critérios obrigatórios da } \\
\text { informação nutricional }\end{array}$} & \multicolumn{7}{|c|}{ Amostras } \\
\cline { 2 - 7 } & A & B & C & D & E & F \\
\hline Porção (g) & C & C & C & C & C & C \\
\hline Valor Energético (kcal) & C & C & C & C & C & C \\
\hline Carboidratos (g) & C & C & C & C & C & C \\
\hline Proteínas (g) & $\mathrm{C}$ & $\mathrm{C}$ & $\mathrm{C}$ & $\mathrm{C}$ & $\mathrm{C}$ & $\mathrm{C}$ \\
\hline Gorduras Totais (g) & $\mathrm{C}$ & $\mathrm{C}$ & $\mathrm{C}$ & $\mathrm{C}$ & $\mathrm{C}$ & $\mathrm{C}$ \\
\hline Gorduras Saturadas (g) & $\mathrm{C}$ & $\mathrm{C}$ & $\mathrm{C}$ & $\mathrm{C}$ & $\mathrm{C}$ & $\mathrm{C}$ \\
\hline Gorduras Trans (g) & $\mathrm{C}$ & $\mathrm{C}$ & $\mathrm{C}$ & $\mathrm{C}$ & $\mathrm{C}$ & $\mathrm{C}$ \\
\hline Fibra Alimentar (g) & $\mathrm{C}$ & $\mathrm{C}$ & $\mathrm{C}$ & $\mathrm{C}$ & $\mathrm{C}$ & $\mathrm{C}$ \\
\hline Sódio (mg) & $\mathrm{C}$ & $\mathrm{C}$ & $\mathrm{C}$ & $\mathrm{C}$ & $\mathrm{C}$ & $\mathrm{C}$ \\
\hline
\end{tabular}

Notas: $\mathrm{C}=$ Conforme; $\mathrm{g}=$ grama; $\mathrm{mg}=$ miligrama; $\mathrm{kcal}=$ quilocaloria.

Fonte: os autores.

Conforme a RDC $\mathrm{n}^{\circ}$ 259/2002, o lote é considerado um item obrigatório nos rótulos de qualquer tipo de produto alimentício. O lote do produto permite que seja realizada a rastreabilidade daquele alimento, possibilitando obter toda e qualquer informação referente às matérias-primas, insumos e materiais de embalagens utilizadas na sua fabricação. Além disso, também é essencial para identificar os consumidores e/ou clientes que possam ter adquirido o lote rastreado (LOPES et al., 2019). Além do lote, outras informações precisam estar descritas nos rótulos, como a lista de ingredientes, declaração de aditivo (se houver), identificação de origem, prazo de validade, preparo e instruções de uso, conservação do produto e tamanho da letra, sendo todos também exigidos pela RDC $n^{\circ} 259 / 2002$, as quais devem se apresentar de forma clara, a fim de facilitar o seu entendimento (BRASIL, 2002). A obrigatoriedade dessas informações é assegurada principalmente pelas RDCs, que impõem aos produtores a presença dos rótulos nos alimentos e as informações necessárias nos mesmos (FEITOZA et al., 2020), de forma a transmitir segurança alimentar e nutricional aos consumidores, promovendo a saúde pública (ARAÚJO, 2017).

A caracterização da informação nutricional dos rótulos das amostras de leite em pó integral analisados segundo os critérios obrigatórios da RDC no 360/2003 e RDC n ${ }^{\circ} 359 / 2003$, em valores de média e desvio padrão, no presente estudo, apresentaram um baixo desvio padrão, caracterizando valores homogêneos em todos os rótulos analisados. A informação nutricional de um alimento é de grande importância para os consumidores, principalmente para a parte da população que busca uma alimentação saudável ou que possua alguma restrição alimentar em decorrência de algum problema de saúde (GREGÓRIO et al., 2020). Assim, o baixo desvio padrão das médias dos valores da informação nutricional encontrado no atual estudo se dá devido à padronização da porção do leite em pó e do valor energético médio de $125 \mathrm{kcal}$ por porção constante no nível IV do grupo do leite e derivados da RDC nº 359/2003 (BRASIL, 2003a).

Ainda, no presente estudo, todas as amostras de leite em pó integral analisadas apresentaram conformidade quanto à rotulagem nutricional obrigatória e a apresentação das expressões dos seus valores exigidas pela $\operatorname{RDC} n^{\circ} 360 / 2003$. A porção para o leite em pó na $\operatorname{RDC~} n^{\circ} 359 / 2003$ é a quantidade suficiente para preparar $200 \mathrm{~mL}$ e a medida caseira é X colheres que correspondam à quantidade de pó para preparar os $200 \mathrm{~mL}$. Nos rótulos das amostras dos leites em pó analisados no 
presente estudo, o modo de preparo para $200 \mathrm{~mL}$ era equivalente a 1 colher e $1 / 2$ de sopa $(26 \mathrm{~g})$, estando de acordo com a legislação (BRASIL, 2003a). No estudo realizado por Gregório et al. (2020) também foram observadas conformidades nos rótulos de leite em pó analisados, onde todas as marcas apresentaram a medida caseira e a porção de referência conforme a RDC nº 359/2003.

Além da porção, o valor energético, de carboidratos, proteínas, gorduras totais, gorduras saturadas, gorduras trans, fibra alimentar e sódio, constantes nos rótulos nutricionais das amostras de leite em pó integral analisadas no atual estudo apresentaram conformidade com a informação nutricional obrigatória exigida pela $\mathrm{RDC} \mathrm{n}^{\circ} 360 / 2003$, corroborando com a análise realizada por Silva Filho, Araújo e Morais (2015), com 30 amostras de leite UHT, sendo 15 integrais, 11 desnatados, 3 semidesnatados e 1 semidesnatado sem lactose, totalizando dezoito marcas, onde todas as amostras avaliadas também estavam de acordo com RDC no 360/2003 quanto a rotulagem nutricional. Já, no estudo realizado por Fernades et al. (2017), foram analisados os rótulos de 10 amostras de leite em pó integral da cidade de Pau dos Ferros-RN, no qual duas das dez amostras analisadas não apresentaram nos rótulos o valor da Gordura Trans, sendo que é um dos itens obrigatórios.

A mudança na rotina das pessoas fez com que a busca por alimentos que já estejam prépreparados ou prontos para consumo aumentasse. Entretanto, esses alimentos são ultraprocessados e normalmente possuem elevada quantidade de gorduras totais e sódio, além de baixo teor de fibras (BRASIL, 2014). A tabela de informação nutricional é um item obrigatório na rotulagem dos alimentos, cujo principal objetivo é auxiliar os consumidores para que possam fazer a melhor escolha para uma alimentação equilibrada, evitando a ocorrência de doenças decorrentes de maus hábitos alimentares, como obesidade, hipercolesterolemias, doenças cardiovasculares e até alguns tipos de câncer. Por isso, essas informações devem ser descritas de forma clara para não induzir o consumidor ao erro ou dúvidas, e sim, facilitar na escolha de produtos específicos, especialmente para aquelas pessoas que possuem restrições alimentares (LIMA et al., 2020).

Nas expressões dos valores nutricionais das amostras de leite em pó avaliadas no presente estudo, os valores energéticos e sódio foram declarados com números inteiros e com três cifras, pois são maiores ou iguais a 100. Os Carboidratos foram declarados com números inteiros e duas cifras, pois são menores que 100 e maiores ou iguais a 10. Já as Proteínas, Gorduras Totais e as Gorduras Saturadas, foram declaradas com uma cifra decimal, pois apresentaram valores menores que $10 \mathrm{e}$ maiores ou iguais a 1. A Gordura Trans foi declarada como zero, portanto os valores foram menores que 0,2g e as Fibras Alimentares, também, foram declaradas como zero, sendo menores que 0,5g, estando todos os valores expressados em conformidade com os critérios da RDC $n^{\circ} 360 / 2003$. No estudo realizado por Gregório et al. (2020) todas as expressões de valores apresentados nos rótulos das amostras analisadas também estavam em conformidade com a RDC $n^{\circ} 360 / 2003$. Já no estudo de Silva et al. (2017) foi possível visualizar que várias amostras que apresentaram valores menores que 10 e maiores que 1 , deveriam apresentar uma cifra decimal, porém apresentaram somente o número inteiro, como foi no caso das Proteínas que foram declaradas em números inteiros, não atendendo aos critérios da legislação.

Diante do exposto, é crescente a busca por alimentos pré-preparados ou prontos para consumo e muitos são os fatores que determinam se um alimento é saudável ou não para o consumidor, sendo um deles as informações contidas na sua rotulagem. Portanto, apesar de, no presente estudo, felizmente, a rotulagem dos leites em pó integral analisadas terem se apresentado em conformidade com as legislações vigentes, tanto para rotulagem geral quanto para a rotulagem nutricional, observou-se que, em outros estudos, a rotulagem destes alimentos não atingiu a totalidade da conformidade com os critérios exigidos pela previsão legal. Isso mostra a carência de fiscalização pelos órgãos competentes, a falta de instrução técnica por parte dos fabricantes de alimentos que os impede de regularizar os rótulos dos seus alimentos antes de se serem comercializados ou até mesmo a negligência de algumas informações importantes ao consumidor por parte do produtor. Cabe ainda ressaltar que os rótulos dos alimentos ainda constituem o único canal de comunicação entre o produto 
e o consumidor, por isso a necessidade do atendimento às exigências das legislações brasileiras vigentes.

\section{CONCLUSÃO}

O presente estudo permitiu concluir que, após realizar a análise das rotulagens de leite em pó integral das sete diferentes marcas comercializadas nos supermercados da cidade do Vale do TaquariRS, todas se apresentaram de acordo com as legislações que regulamentam a rotulagem de alimentos no Brasil. Ainda, foi possível demonstrar que as indústrias desta região estão seguindo corretamente as normas e especificações que a legislações brasileiras vigentes impõem quanto aos critérios de rotulagem de alimentos.

Além dos resultados positivos deste trabalho, cabe aos órgãos que regulamentam essas especificações continuarem a fiscalizar as indústrias para que erros não sejam cometidos, prezando pela saúde do consumidor final.

\section{REFERÊNCIAS}

\section{ASSOCIAÇÃO BRASILEIRA DA INDÚSTRIA DE ALIMENTOS. Relatório anual, 2019.}

AGÊNCIA NACIONAL DE VIGILÂNCIA SANITÁRIA. Anvisa aprova norma sobre rotulagem nutricional, 2020.

ARAÚJO, W. D. R. Importância, estrutura e legislação da rotulagem geral e nutricional de alimentos industrializados no brasil. Revista Acadêmica Conecta FASF, v. 2, n. 1, p. 35-50, 2017.

BRASIL. Agência Nacional de Vigilância Sanitária. Resolução RDC no 259, de 20 de setembro de 2002. Brasília: Agência Nacional de Vigilância Sanitária, 2002.

BRASIL. Agência Nacional de Vigilância Sanitária. Resolução RDC no 359, de 23 de dezembro de 2003. Brasília: Agência Nacional de Vigilância Sanitária, 2003a.

BRASIL. Agência Nacional de Vigilância Sanitária. Resolução RDC no 360, de 23 de dezembro de 2003. Brasília: Agência Nacional de Vigilância Sanitária, 2003 b.

BRASIL. Ministério da Agricultura, Pecuária e Abastecimento. Instrução normativa $\mathbf{n}^{\mathbf{0}} \mathbf{5 3}$, de $1^{\circ}$ de outubro de 2018. Brasília: Ministério da Agricultura, Pecuária e Abastecimento, 2018.

BRASIL. Ministério da Saúde. Secretaria de Atenção à Saúde. Departamento de Atenção Básica. Guia alimentar para a população brasileira. Ministério da Saúde, Secretaria de Atenção à Saúde, Departamento de Atenção Básica. 2. ed., 1. reimpr. Brasília: Ministério da Saúde, 2014. 156 p.

FEITOZA, J. V. F. et al. Avaliação da rotulagem dos alimentos comercializados no município de Apodi - RN. Revista Brasileira de Gestão Ambiental, v. 14, n. 1, p. 28-32, 2020.

FERNADES, A. V. et al. Avaliação da rotulagem de leite em pó comercializado na cidade de Pau dos Ferros-RN. Revista Brasileira de Agrotecnologia, v. 7, n. 1, p. 38-41, 2017.

GREGÓRIO, M. G. et al. Análise da rotulagem de diferentes marcas de leite em pó integral comercializados na Cidade de Acari, Rio Grande do Norte. Research, Society and Development, v. 9, n. 8, e860986183, 2020. 
INSTITUTO BRASILEIRO DE GEOGRAFIA E ESTATÍSTICA. Censo 2021: Rebanho bovino tem leve alta em 2019, após dois anos seguidos de quedas, 2020.

IWASE, C. H. T. Bacillus cereus e esporos de bacilos aeróbios mesófilos e termófilos deteriorantes em leite em pó. 2017. 83f. Dissertação (Mestrado em Ciência de Alimentos) Universidade Estadual de Campinas, Campinas, 2017.

LIMA, A. B. et al. Comportamento do consumidor frente à informação nutricional em rotulagem de produtos alimentícios. ConnectionLine - Revista Eletrônica do Univag, n. 22, p. 48-63, 2020.

LOPES, L. O. et al. Rastreabilidade: aspectos regulatórios e aplicação no processamento de leite e derivados. 2019.

OLIVEIRA, L. S.; MARCHIORE, N. G. Caracterização da produção do leite em pó e análise da forma de secagem: uma revisão. 2017. In: Mostra Científica de Alimentos, 3, 2017, Medianeira. Anais... Medianeira: UTRPR, 2017.

SANTOS, E. N. et al. Avaliação da Rotulagem de produtos de origem animal comercializados na cidade de Santa Luzia-PB. Revista Brasileira de Gestão Ambiental, v. 14, n. 1, p. 6-11, 2020.

SILVA FILHO, C. R. M.; ARAÚJO, C. D. L.; MORAIS, W. S. Análise da rotulagem de leites UHT comercializados na cidade de João Pessoa-Pb. In: Simpósio de Segurança Alimentar: Alimentação e Saúde, 5, 2015, Bento Gonçalves. Anais... Bento Gonçalves: SBCTA-RS, 2015.

SILVA, M. C. et al. Avaliação de rótulos de diferentes marcas de leite em pó integral comercializados na cidade de Garanhuns - PE. In: Congresso Internacional das Ciências Agrárias. 2, 2017, Recife. Anais... Recife: Cointer DPVAgro, 2017.

SIQUEIRA, K. B. O Mercado Consumidor de Leite e Derivados. Circular técnica 120. Juiz de Fora: EMBRAPA, 2019. 